Sozial-ökologische Probleme aus ordonomischer Perspektive

\title{
Social Entrepreneurship und Ordnungspolitik
}

\begin{abstract}
Soziale Unternehmer können als Change Agents zur Lösung gegenwärtiger Herausforderungen beitragen und Ordnungspolitik kann durch geeignete Regeln für die Wirtschaft zum Erreichen gesellschaftlicher Ziele beitragen. Im Rahmen einer Kombination beider Ansätze erscheinen Social Entrepreneurship und Ordnungspolitik als komplementäre Elemente einer Governance für Nachhaltigkeit. Von Stefan Hielscher und Markus Beckmann
\end{abstract}

K imaschutz, Erhaltung der Biodiversität, Naturschutz, Kinderarbeit, extreme Armut, Bildungsdefizite, demografischer Wandel: Die entstehende Weltgesellschaft steht vor einer Reihe ökologischer und sozialer Ordnungsprobleme, die bis dato weder durch marktliche Organisation noch durch staatliche Koordination zufriedenstellend gelöst werden können (1).

\section{Ordonomik als Lösungsansatz}

Social Entrepreneurship und Ordnungspolitik sind zwei Forschungsfelder, die sich um einen theoretischen Beitrag zur Lösung dieser Probleme bemühen. Social Entrepreneurship ist eine sehr junge und dynamische Disziplin. Sie fragt: Wie können „Social Entrepreneurs“ oder "Soziale Unternehmer“ als Change Agents im Auftrag ökologischer Problembearbeitung und gesellschaftlicher Wertschöpfung zur Lösung dieser Herausforderungen beitragen? Auf diese Frage entwickelt die Literatur Antworten, bei der insbesondere die Rolle von Führungspersönlichkeit, innovative Geschäftsmodelle und Best Practices im Mittelpunkt stehen. Der Fokus liegt auf der Handlungsebene individueller Akteure.

Die Ordnungspolitik hingegen ist ein traditionsreiches Forschungsprogramm mit wirtschafts- und gesellschaftstheoretischer Ausrichtung. Sie fragt: Wie kann die Politik durch geeignete Regelsetzung die Wettbewerbsordnung der Wirtschaft so in Kraft setzen und institutionell absichern, dass die gewünschten gesellschaftlichen, also die sozialen, ökologischen und moralischen, Ziele bestmöglich erreicht werden? Der Fokus der ordnungspolitischen Fragestellung liegt demnach auf der Ordnungsebene der Regeln, und zwar für die Wirtschaft ebenso wie für die Gesellschaft.

Wie können diese unterschiedlich ansetzenden Fragestellungen problemorientiert zusammengeführt werden? Eine Antwort auf diese Frage kann mithilfe eines Ansatzes entwickelt werden, der unter dem Stichwort Ordonomik diskutiert wird (2). Die Ordonomik setzt auf ein rational-choice-orientiertes Forschungsprogramm zur Analyse von Sozialstruktur und Semantik.

\section{Sozialstruktur}

Die Ordonomik rekonstruiert ökologische und gesellschaftliche Probleme als soziale Dilemmata. In Dilemmastrukturen kommt es zu kollektiver Selbstschädigung. Die beteiligten Akteure bleiben unter ihren Möglichkeiten, weil jeder fürchten muss, dass die eigenen Vorleistungen von potenziellen Kooperationspartnern ausgebeutet werden. Die Ordonomik unterscheidet einseitige und mehrseitige Dilemmata. Der erste Fall erfordert eine individuelle Bindung, um produktive Gegenleistungen bei den Kooperationspartnern auszulösen. Der zweite Fall, das genuine Gefangenendilemma, erfordert eine kollektive Bindung aller beteiligten
Akteure, um kooperative Vorleistungen vor Ausbeutung zu schützen. Abbildung 1 illustriert an je zwei historischen und zwei aktuellen Beispielen, wie Social Entrepreneurs Bindungen zur Überwindung sozialer Dilemmata einsetzen können.

\section{Überwindung sozialer Dilemmata}

Eine schnell wachsende Bevölkerung und eine unterentwickelte medizinische Infrastruktur führten in der zweiten Hälfte des 19.Jahrhunderts in der Industriestadt Essen zur Ausbreitung von Seuchen und Epidemien. Um mithilfe von Ärzten und Krankenhäusern die erforderliche Gesundheitsvorsorge zu gewährleisten, führte der Pionierunternehmer Alfred Krupp bereits 1853 eine betriebliche Krankenversicherung ein. Durch diese Organisation kollektiven Handelns für seine Mitarbeiter gelang es ihm, Infektionskrankheiten bereits früh einzudämmen und teure Produktionsausfälle zu verhindern (Quadrant I der Abbildung).

In den 1850er Jahren bestand ein Gros der kruppschen Belegschaft aus Wanderarbeitern. Für die Herstellung von Qualitätsprodukten benötigte Krupp jedoch eine sesshafte Stammbelegschaft, die bereit war, spezifische Investitionen in die Fabrik einzugehen. Krupp reagierte auf dieses Problem mit einer individuellen Selbstbindung, indem er seinen Arbeitern höhere Löhne als bei der Konkurrenz in Aussicht stellte und zusätzlich die Glaubwürdigkeit dieses Versprechens durch betriebliche Sozialpolitik wie Wohnungsbau, Konsumanstalt, Schulen und Kindergärten sicherstellte (Quadrant III der Abbildung).

Ein gravierendes aktuelles Armutsproblem in Entwicklungsländern ist das Problem, dass Kreditnehmer, vor allem im ländlichen Raum, in der Regel nicht über Kreditsicherheiten verfügen. Ein Kreditgeber muss daher fürchten, dass ein Kredit nicht zurückgezahlt wird, sodass viele Kreditgeschäfte erst gar nicht zustande kommen. Mikrokredit-Programme lösen dieses Problem, in dem sie dem Kreditnehmer helfen, eine individuelle Selbstbindung einzugehen, durch die er sich 
Abbildung 1: Bindungsmatrix für Social Entrepreneurship

\begin{tabular}{|c|c|c|c|}
\hline & & \multicolumn{2}{|l|}{ Bindungsform } \\
\hline & & Unternehmen & Stakeholder \\
\hline & 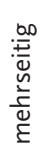 & $\begin{array}{l}\text { (IV) } \\
\text { Kollektive } \\
\text { Selbstbindung } \\
\text { (Standards, FSC) }\end{array}$ & $\begin{array}{l}\text { Service } \\
\text { für kollektive } \\
\text { Selbstbindung } \\
\text { (Kruppsche } \\
\text { Krankenversicherung) }\end{array}$ \\
\hline $\begin{array}{l}\text { Dilemma- } \\
\text { Struktur }\end{array}$ & 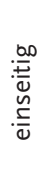 & $\begin{array}{l}\text { (III) } \\
\text { Individuelle } \\
\text { Selbstbindung } \\
\text { (Kruppsche Lohnbindung } \\
\text { und betriebl. Sozialpolitik) }\end{array}$ & $\begin{array}{l}\text { Service } \\
\text { für individuelle } \\
\text { Selbstbindung } \\
\text { (Mikrokredite) }\end{array}$ \\
\hline
\end{tabular}

Quelle: Hielscher / Pies / Beckmann (2007)

glaubhaft zur Rückzahlung des Kredits verpflichten kann. Konkret wird Glaubwürdigkeit etwa durch sozialen Gruppendruck gewährleistet, beispielsweise indem ein Kreditring gebildet wird, in dem sich mehrere Kreditnehmer der Dorfgemeinschaft wechselseitig kontrollieren (Quadrant II der Abbildung).

Existenzielle Armuts- und Ernährungsprobleme führen in Entwicklungsländern häufig zu einem schonungslosen Umgang mit Naturressourcen, insbesondere mit Wäldern, mit gravierenden Folgen unter anderem für das weltweite Klima. Dieses Problem ist darauf zurückzuführen, dass natürliche Ressourcen keinen oder einen zu niedrigen Preis haben, sodass Unternehmen im marktlichen Wettbewerb durch Ressourcenschonung weder Umsätze erwirtschaften noch Kosten sparen können. Unternehmen können allerdings dazu beitragen, dass Umweltschutz zu wirtschaftlichem Erfolg im Markt führt, zum Beispiel indem sie sich freiwillig und glaubwürdig zu ökologischen Standards verpflichten. Ein Beispiel dafür ist das kollektive Branchenlabel Forest Stewardship Council (FSC). Auf diese Weise kann es ihnen gelingen, gemeinschaftlich eine höhere Zahlungsbereitschaft für ihre Produkte zu aktivieren und sich gleichzeitig vor möglichen Trittbrettfahrern zu schützen. (Quadrant IV der Abbildung).

In allen vier Beispielen wirken korporative Akteure an einer nachhaltigen Lösung ökologischer und sozialer Probleme mit, indem sie ein Selbstbindungspotenzial für sich und andere auf- bauen und unternehmerisch einsetzen, um soziale Dilemmata $\mathrm{zu}$ überwinden. Diese Perspektive ermöglicht interessante Anschlussüberlegungen für das Verständnis sowohl von Social Entrepreneurship als auch von Ordnungspolitik.

\section{Semantik}

Die Funktion innovativer Change Agents im Auftrag ökologischer Problemlösung und gesellschaftlicher Wertschöpfung entfalten Social Entrepreneurs vor allem dann, wenn sie nicht nur ihre individuellen Spielzüge zu optimieren wissen, sondern wenn sie Regelinnovationen leisten, die zur Weiterentwicklung wechselseitig vorteilhafter Spielregeln beitragen. Aus ordonomischer Perspektive liegt die Raison d'être von Social Entrepreneurship darin, das Selbstbindungspotenzial korporativer Akteure zur Lösung bisher nicht überwundener Ordnungsprobleme einzusetzen. Und zwar nicht nur im Notfor-Profit-Bereich, sondern gerade auch im marktlich-wettbewerblichen Kontext.

Aus traditioneller ordnungspolitischer Sicht ist jedoch die Überwindung sozialer Dilemmastrukturen die exklusive Aufgabe des Staates. Der Staat setzt die Spielregeln; die wirtschaftlichen und gesellschaftlichen Akteure optimieren dann ihre Spielzüge. Dieser strikten Trennung liegt die Überlegung zugrunde, dass insbesondere die Einflussnahme wirtschaftlicher Akteure auf die Regelsetzung die Gefahr des Rent Seeking und marktgefährdender Kartelle beinhaltet. Die Beispiele zeigen jedoch, dass private Akteure Lücken nationalstaatlicher Regelsetzung auffüllen können, um Märkte nicht außer Kraft, sondern um sie gerade in Kraft zu setzen. Angesichts drängender ökologischer und sozialer Governance-Probleme verbindet sich für die Ordnungstheorie die Aufgabe, eine tragfähige
Theorie von New Governance zu entwickeln, die nicht nur staatliche Akteure, sondern auch unternehmerisch und zivilgesellschaftlich agierende Social Entrepreneurs als Regelsubjekte adressiert.

Die Verwirklichung von Nachhaltigkeit durch Markt und Staat ist somit als wechselseitiger Lernprozess für Governance zu denken, in den einerseits Unternehmen genuine Ordnungsinnovationen einbringen können und in dem andererseits staatliche Rahmensetzung Anreize für Social Entrepreneurship zu schaffen vermag.

\section{Literatur}

Hielscher, S. / Beckmann, M.: Social Entrepreneurship und Ordnungspolitik: Zur Rolle gesellschaftlicher Change Agents am Beispiel des Kruppschen Wohlfahrtsprogramms. Diskussionspapier Nr. 2008-5 des Lehrstuhls für Wirtschaftsethik an der Martin-Luther-Universität Halle-Wittenberg, 2008.

Hielscher, S. / Pies, I. / Beckmann, M.: Wie können Corporate Citizens voneinander lernen? Anregungen für den Global Compact der Vereinten Nationen, Diskussionspapier Nr. 2007 13 des Lehrstuhls für Wirtschaftsethik an der Martin-Luther-Universität Halle-Wittenberg, 2007.

Pies, I.: Markt vs. Staat? Über Denk- und Handlungsblockaden im Zeitalter der Globalisierung. In: Graf Ballestrem, K. / Gerhardt, V. / Ottmann, K. / Thompson, M.P. / Zehnpfennig, B. (Hrsg.): Politisches Denken. Jahrbuch 2006/2007. Berlin 2007, S. 259-293

Pies, I. / Beckmann, M. / Hielscher, S.: Mind the Gap! Ordonomische Überlegungen zur Sozialstruktur und Semantik moderner Governance. Diskussionspapier Nr. 2007-16 des Lehrstuhls für Wirtschaftsethik an der Martin-Luther-Universität Halle-Wittenberg, 2007.

\section{Anmerkungen}

(1) Dieser Beitrag referiert thesenartig zentrale Gedanken aus Hielscher/Beckmann 2008.

(2) Vgl. hierzu Pies 2007 sowie

Pies/Beckmann/Hielscher 2007.

\section{AUTOREN + KONTAKT}

Stefan Hielscher ist Mitarbeiter und Markus Beckmann ist Doktorand am Lehrstuhl für Wirtschaftsethik der Martin-LutherUniversität Halle-Wittenberg.

Martin-Luther-Universität Halle-Wittenberg, Lehrstuhl für Wirtschaftsethik, Große Steinstraße 73, 06108 Halle. Tel.: +49 345 552-3387,

E-Mail: stefan.hielscher@wiwi.uni-halle.de; markus.beckmann@wiwi.uni-halle.de 
(c) 20I0 Authors; licensee IÖW and oekom verlag. This is an article distributed under the terms of the Creative Commons Attribution Non-Commercial No Derivates License (http://creativecommons.org/licenses/by-nc-nd/3.o/), which permits unrestricted use, distribution, and reproduction in any medium, provided the original work is properly cited. 